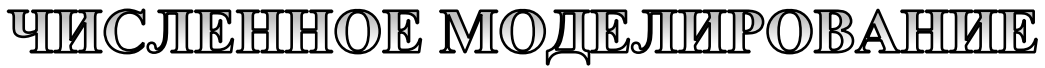

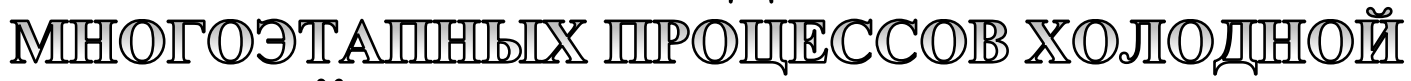

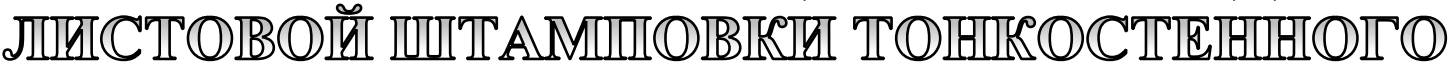

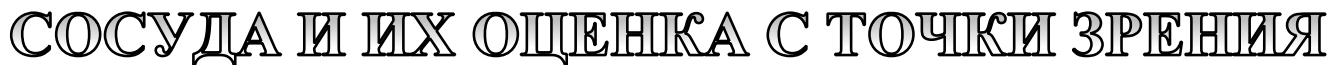

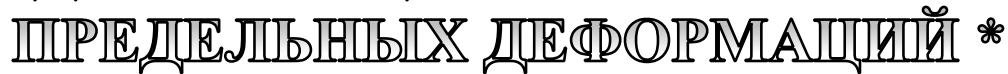

И.Э. Келлер, Институт механики сплошных сред УрО РАН;

Пермский национальный исследовательский политехнический университет

А.В. Казанцев, Пермский национальный исследовательский политехнический университет

А.А. Адамов, Институт механики сплошных сред УрО РАН

Д.С. Петухов, Институт механики сплошных сред УрО РАН;

Пермский национальный исследовательский политехнический университет

В.Н. Трофимов, Пермский национальный исследовательский политехнический университет

А.Н. Оборин, Лысьвенский завод эмалированной посуды

С.Б. Чугайнов, Лысьвенский завод бытовой техники

\section{Для цитирования:}

Келлер И.Э., Казанцев А.В., Адамов А.А., Петухов Д.С., Трофимов В.Н., Оборин А.Н., Чугайнов С.Б. Численное моделирование многоэтапных процессов холодной листовой штамповки тонкостенного сосуда и их оценка с точки зрения предельных деформаций // Вестник Пермского федерального исследовательского центра. - 2021. - № 2. - C. 48-60. https://doi.org/10.7242/2658-705X/2021.2.5

Выполнена оценка многоэтапного процесса холодной штамповки тонкостенного стального сосуда с учетом технологической наследственности. Качество изделия связывается с остаточным ресурсом пластичности, то есть с удаленностью его деформированного состояния от кривой предельных деформаций. Для расчета процесса используется модель больших пластических деформаций анизотропной оболочки, учитывающая динамику и контактное взаимодействие с инструментом, численно реализованная в пакете LS-DYNA ${ }^{\circledR}$. Использованы имеющиеся в библиотеке пакета закон пластического течения анизотропного листа, ассоциированный с функцией текучести Барлата Yld 2 000-2d со степенным изотропным деформационным упрочнением, потенциал Пенга - Ландела нелинейно-упругого поведения полиуретанового штампа и закон трения Кулона для описания контактного взаимодействия изделия с инструментом.

Материальные константы малоуглеродистой листовой стали DC04EK толщиной 0,7 мм и полиуретана СКУ-ПФЛ определены ранее по данным экспериментов. Кривая предельных деформаций построена по искажению координатной сетки вблизи зон локализации деформации и разрушения

\footnotetext{
* Работа выполнена при финансовой поддержке гранта совместного конкурса РФФИ и правительства Пермского края (проект № 17-48-590310p-a). Авторы признательны Н.Л. Русановой (ООО УК «Лысьвенские заводы») за организацию совместных исследований .
} 
сосуда в технологическом процессе, из которого исключен промежуточный отжиг, и по результатам теста на разрушение при одноосном растяжении.

Исследуются особенности траекторий деформации в контрольных точках боковой поверхности изделия на каждом этапе технологического процесса, состоящего из последовательности операций вытяжки, раздачи и ссаживания. Расчеты траекторий подтверждены экспериментом на прессовом оборудовании, используемом в качестве испытательного. Установлено, что операция раздачи заготовки после ее вытяжки быстро приближает материал к предельному состоянию и требует предварительного восстановления ресурса пластичности путем отжига. Отмечается перспективность технологий штамповки с меньшими степенями раздачи и с большими степенями ссаживания, способных сохранять ресурс пластичности без промежуточного отжига.

Ключевые слова: многоэтапный технологический прочесс, холодная листовая штамповка, численный расчет, анизотропная пластичность, предельные деформации, малоуглеродистая сталь, идентификация свойств материала, экспериментальное подтверждение.

\section{Введение}

Производство металлических тонкостенных сосудов холодной листовой штамповкой предусматривает ряд последовательных операций, оптимизированных с целью предотвращения образования дефектов изделия. Существующая технология штамповки стального эмалированного чайника и некоторых других подобных изделий в АО «Лысьвенский завод эмалированной посуды» (г. Лысьва Пермского края) не может обойтись без промежуточного восстановления ресурса пластичности заготовки рекристаллизационным отжигом. Данная операция усложняет и удорожает производство изделия, наносит вред экологии из-за необходимости травления кислотой окалины отожженной заготовки. Разработка альтернативного технологического процесса, оптимального с точки зрения себестоимости и качества изделия, в современных условиях практически немыслима без численного моделирования. Для этого необходима методика, включающая математическую модель пластичности и разрушения материала вместе с процедурой идентификации ее констант, численный расчет процесса с использованием этой модели и экспериментальную верификацию полученных результатов.
Вопросам разработки подобной методики посвящены монографии [1-2], обзоры [3-5], оригинальные статьи [6-9]. Для численного решения геометрически и физически нелинейных задач листовой штамповки, с характерными для них протяженными поверхностями контакта заготовки и инструмента, подходят универсальные и специализированные коммерческие пакеты программ на основе метода конечных элементов в сочетании с явным методом интегрирования уравнений по времени. Как правило, такие пакеты имеют обширные библиотеки моделей материала, на которые при решении задач стоит рассчитывать в первую очередь.

Исследуемые технологические процессы производства тонкостенных сосудов на Лысьвенских заводах содержат важную операцию ссаживания (редукции диаметра) осесимметричной заготовки обкаткой роликом на оправке. Операция сопровождается высокоскоростным деформированием металла, может порождать динамические моды образования дефектов и потому требует динамической постановки. Все это определило выбор для численного расчета исследуемого процесса пакета LS-DYNA ${ }^{\circledR}$.

Постановка задачи. Процесс штамповки чайника включает последовательные этапы (схематически изображенные 
на рис. 1): I, II, III - вытяжку стакана с формованием дна, IV - раздачу полиуретановым штампом, V-ссаживание горловины. Размеры исходной и промежуточных заготовок приведены на рис. 2,a. На рис. 2,б приведена сетка материальных координат, наносимая на недеформированную заготовку-круг лазерной гравировкой. Контрольные точки располагаются на пересечении координат. Эти же точки рассматриваются в численном расчете для сопоставления его результатов с экспериментом. На этапах I, II, III могут появляться гофры на краях и в верхней части заготовок-стаканов, а на этапе $\mathrm{V}-$ недопустимые утонения стенки в местах перехода диаметров в области горловины.
Основные дефекты - полосы сдвига и трещины, образуются в зонах предельных деформаций на боковой поверхности заготовки (рис. 3) на этапе IV без предварительного рекристаллизационного отжига. Исследуются закономерности возникновения и наследования перечисленных дефектов на каждом из этапов технологического процесса.

История изменения состояния материала в контрольных точках боковой поверхности осесимметричного сосуда и их близость к предельным состояниям отражаются на диаграмме предельных деформаций - части плоскости главных деформаций оболочки вдоль продольной $\left(\varepsilon_{1} \geq 0\right)$ и окружной $\left(\varepsilon_{2} \leq 0\right)$ координат.
I

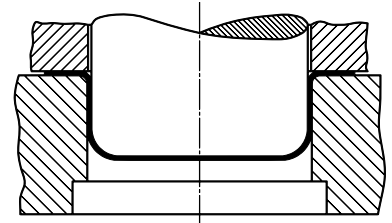

II

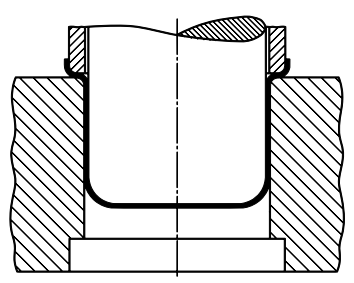

III

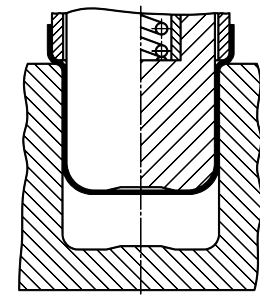

IV
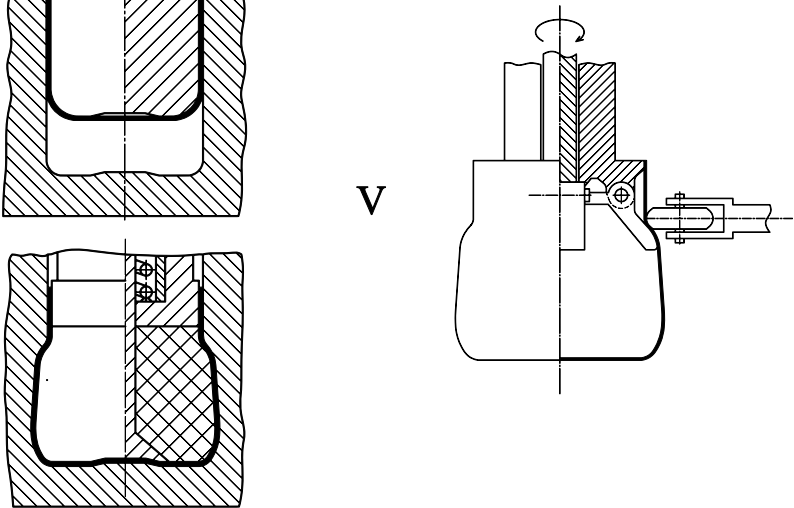

Рис. 1. Этапы технологического прочесса производства чайника

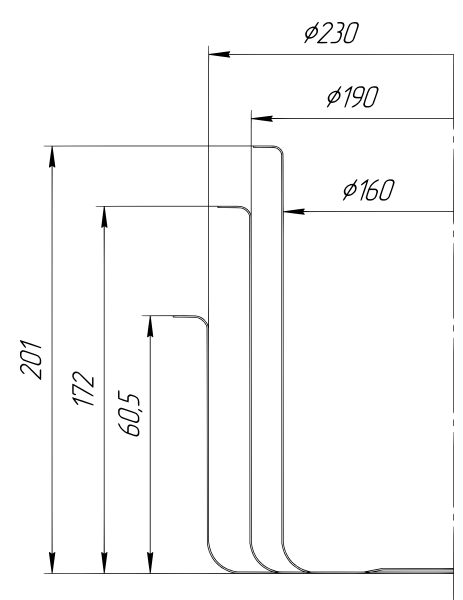

$a$

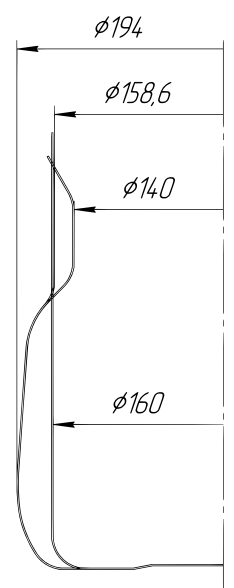

(emка (видимая гравиров̈ка)

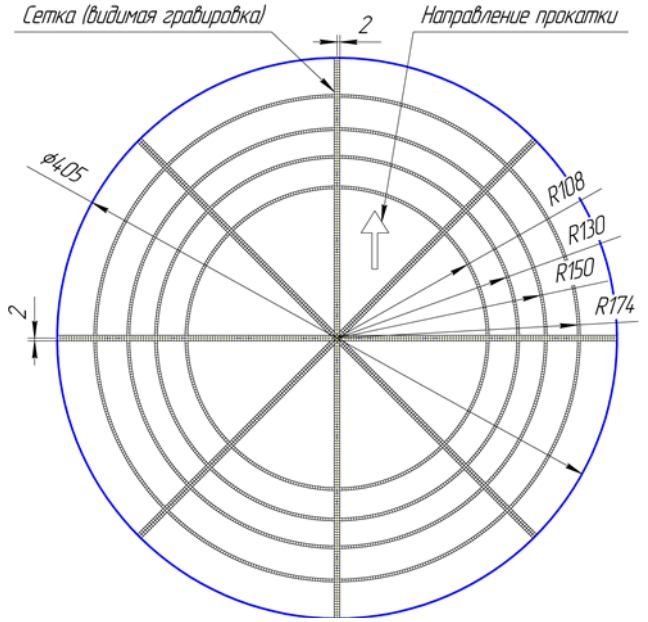

$\sigma$

Рис. 2. Размеры заготовок (а) и сетка материальных координат на недеформированной заготовке (б) 


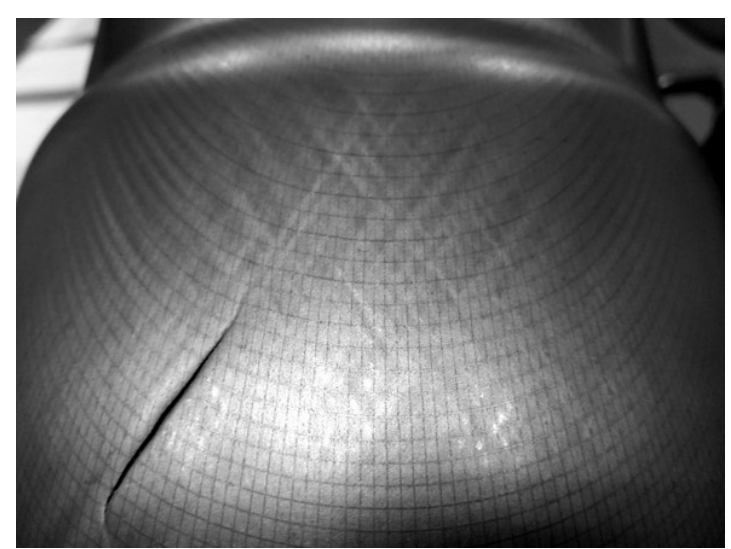

Рис. 3. Деформированная координатная сетка в зоне предельных деформаций

Оставшаяся за пределами данной области часть диаграммы предельных деформаций отображает состояния в точках дна сосуда, далекие от предельного.

Для восстановления ресурса пластичности после этапов вытяжки существующий процесс предусматривает промежуточный отжиг. Эта операция усложняет процесс технологически и логистически и сопровождается экологически вредной операцией травления отожженной заготовки кислотой. Для проектирования альтернативной технологии производства изделия, исключающей отжиг, необходим критерий качества изделия, учитывающий историю изменения состояния материала в ходе многоэтапного процесса деформирования. С этой целью далее выполняется численный расчет процесса и делается его оценка с точки зрения остаточного ресурса пластичности изделия, в качестве меры которого рассматривается близость деформированного состояния к предельно допустимым. На основе данной оценки даются рекомендации по изменению технологии, позволяющие исключить из нее промежуточный отжиг.

Математическая модель и ее численная реализация. В численном расчете процесса для описания больших пластических и малых упругих деформаций металлов используется стандартная модель [10] с формулировкой в терминах текущего лагранжева подхода в скоростях. Принимается аддитивность упругих и пластических тензоров деформаций скорости, при этом упругий тензор связывается линейно с производной Яуманна тензора напряжений Кирхгоффа, а пластический - ассоциированным законом пластического течения с изотропным упрочнением с материальной производной этого тензора. Поскольку пластические деформации в точках боковой поверхности сосуда на любом этапе технологического процесса на три порядка превосходят упругие, далее в анализе результатов будут фигурировать полные деформации, а среда приближенно полагается несжимаемой. Тогда главная деформация по толщине оболочки $\varepsilon_{3}$ приближенно равна $-\varepsilon_{1}-\varepsilon_{2}$, где $\varepsilon_{1}, \varepsilon_{2}, \varepsilon_{3}$ - главные значения левой логарифмической меры деформации (индекс 1 отвечает продольному, 2 - окружному, а 3 - радиальному направлению цилиндрической системы координат).

Для описания холодного пластического деформирования анизотропной листовой стали используется функция текучести Барлата Yld 2 000-2d [11]

$$
\begin{gathered}
\phi=\phi^{\prime}+\phi^{\prime \prime}=2 \sigma_{0}^{m}, \\
\phi^{\prime}=\left|\chi_{+}^{\prime}-\chi_{-}^{\prime}\right|^{m}, \\
\phi^{\prime \prime}=\left|2 \chi_{-}^{\prime \prime}+\chi_{+}^{\prime \prime}\right|^{m}+\left|2 \chi_{+}^{\prime \prime}+\chi_{-}^{\prime \prime}\right|^{m}, \\
\chi_{ \pm}^{\prime}=(1 / 2)\left(\chi_{11}^{\prime}+\chi_{22}^{\prime} \pm \sqrt{\left(\chi_{11}^{\prime}-\chi_{22}^{\prime}\right)^{2}+4 \chi_{12}^{\prime 2}}\right), \\
\chi_{ \pm}^{\prime \prime}=(1 / 2)\left(\chi_{11}^{\prime \prime}+\chi_{22}^{\prime \prime} \pm \sqrt{\left(\chi_{11}^{\prime \prime}-\chi_{22}^{\prime \prime}\right)^{2}+4 \chi_{12}^{\prime \prime 2}}\right), \\
\left\{\begin{array}{l}
\chi_{11}^{\prime} \\
\chi_{11}^{\prime \prime} \\
\chi_{22}^{\prime \prime} \\
\chi_{12}^{\prime}
\end{array}\right\}=\left[\begin{array}{ccc}
\alpha_{1} & 0 & 0 \\
0 & \alpha_{2} & 0 \\
0 & 0 & \alpha_{7}
\end{array}\right]\left\{\begin{array}{l}
s_{z z} \\
s_{\varphi \varphi} \\
s_{\varphi z}^{\prime}
\end{array}\right\}, \\
{\left[\begin{array}{ccc}
(1 / 3)\left(4 \alpha_{5}-\alpha_{3}\right) & (2 / 3)\left(\alpha_{6}-\alpha_{4}\right) & 0 \\
(2 / 3)\left(\alpha_{3}-\alpha_{5}\right) & (1 / 3)\left(4 \alpha_{4}-\alpha_{6}\right) & 0 \\
0 & 0 & \alpha_{8}
\end{array}\right]\left\{\begin{array}{l}
s_{z z} \\
s_{\varphi \varphi} \\
s_{\varphi z}
\end{array}\right\} .}
\end{gathered}
$$

Здесь $\sigma_{0}=141 \mathrm{MПа} \mathrm{-} \mathrm{предел} \mathrm{текуче-}$ сти в направлении прокатки; $m=6$; $s_{i j}$ - компоненты девиатора тензора напряжений Кирхгоффа; материальные константы функции текучести для холоднокатаной малоуглеродистой листовой стали марки 
DC04EK толщиной 0,7 мм определены в [12]; там же для этой стали найдены константы $\varepsilon_{0}=0,00264, n=0,226$ закона изотропного деформационного упрочнения

$$
s_{u}=\sigma_{0}\left(\varepsilon_{u} / \varepsilon_{0}\right)^{n},
$$

где $s_{u}=\sqrt{3 s_{i j} s_{i j} / 2}-$ интенсивность напряжений, $\varepsilon_{u}=\int_{0}^{t} \sqrt{2 d_{i j} d_{i j} / 3} d t-$ накопленные за время $t$ деформации, $d_{i j}-$ компоненты тензора деформаций скорости. В пакете LS-DYNA $^{\circledR}$ данной модели соответствует стандартный материалу МАT_133 [13].

Таблица 1

Значения констант функции текучести Барлата YId 2000-2d

\begin{tabular}{|c|c|c|c|c|c|c|c|}
\hline$\alpha_{1}$ & $\alpha_{2}$ & $\alpha_{3}$ & $\alpha_{4}$ & $\alpha_{5}$ & $\alpha_{6}$ & $\alpha_{7}$ & $\alpha_{8}$ \\
\hline 1,04 & 0,997 & 0,906 & 0,936 & 0,959 & 0,915 & 0,950 & 0,956 \\
\hline
\end{tabular}

Нелинейно-упругие свойства литьевого полиуретана СКУ-ПФЛ, необходимые для моделирования операции раздачи, описываются потенциалом Пенга - Ландела

$$
\begin{gathered}
\Phi=(1 / 2) \mu\left(I_{1} I_{3}^{-1 / 3}-3\right)+(1 / 2) B \theta^{2}, \\
\theta=I_{3}^{1 / 2}-1,
\end{gathered}
$$

где $I_{1}, I_{3}-$ инварианты меры деформаций Фингера, с константами $\mu=4,44 \mathrm{MПа,}$ $B=2,44$ ГПа, найденными в [14]. Этой модели отвечает стандартный материал MAT_077 пакета LS-DYNA ${ }^{\circledR}[13]$.

Численное решение контактной динамической задачи пластического деформирования оболочки выполнено в пакете LS-DYNA $^{\circledR}$, в котором реализован метод конечных элементов с учетом геометрической нелинейности и в сочетании с явной схемой интегрирования уравнений динамики по времени. Вычислительный эксперимент проводился в соответствии с рекомендациями разработчиков программного обеспечения [15-16] по снижению времени счета путем искусственного увеличения плотности материала заготовки и подбора коэффициентов диссипации кинетической энергии системы для устранения возникающих динамических эффектов. Взято
10 точек интегрирования по толщине оболочечного конечного элемента с формулировкой №16. Использовались контакты *AUTOMATIC_SURFACE_TO_SURFACE с кулоновской моделью статического трения с коэффициентом: 0\% - для контакта металлической заготовки с металлической матрицей, $12 \%$ - для контакта металла с полиуретаном, $8-15 \%$ - для металлической заготовки с металлическим пуансоном. Это соответствует применяемым на производстве смазочным жидкостям для контактов металлических поверхностей.

Численные эксперименты показали, что в указанных границах трение практически не влияет на напряженно-деформированное состояние заготовки. В то же время на устойчивость численного счета при моделировании процесса глубокой вытяжки значительное влияние оказывают технологические зазоры между инструментом и заготовкой. Последние выбирались в диапазоне от 1,25 до 3 толщин заготовки. Несмотря на использование автоматических контактов, затратных с точки зрения вычислительных ресурсов, повышенное число точек интегрирования, выбор решателя двойной вещественной точности и явной схемы интегрирования уравнений движения системы, применение нелинейного анизотропного материала и наличие 50-80 тысяч узлов в конечно-элементной сетке, моделирование на персональном компьютере одного этапа вытяжки или раздачи длилось около 10 минут, а расчет операции ссаживания требовал порядка полутора часов.

Расчет операций штамповки изделия и анализ его состояния. На рис. 4-7 приведены данные численного расчета этапов I-VI рассматриваемого технологического процесса, причем в четвертом этапе не учитывался промежуточный отжиг. В правой части рисунков приводится конфигурация изделия после соответствующего этапа с полем толщин стенки; в левой части представлено деформированное состояние боковой стенки изделия в контрольных точках, как вычисленное, так и определенное в эксперименте 


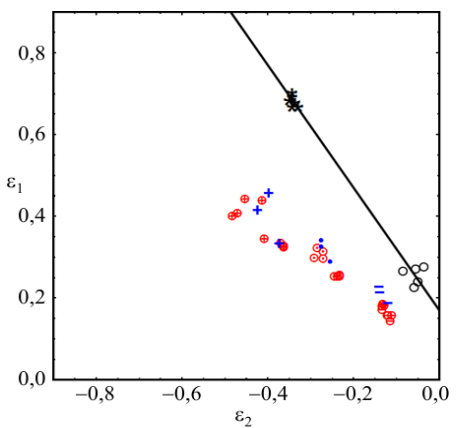

$a$

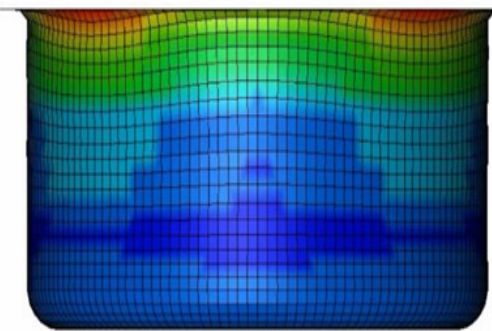

Толщина, мм

1,0430

1,0090

0,9755

0,9419

0,9082

0,8745

0,8408

0,8071

0,7734

0,7397

0,7060

Рис. 4. Деформированное состояние (а) и поле толщин в мм (б) стакана после этапа I

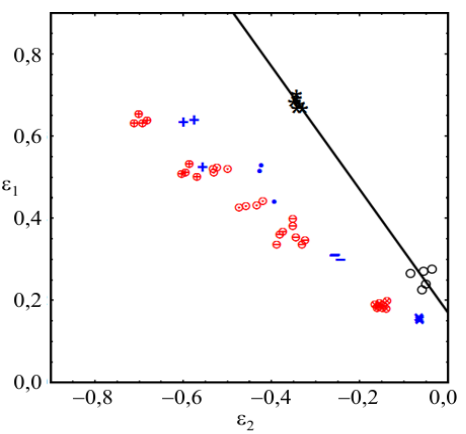

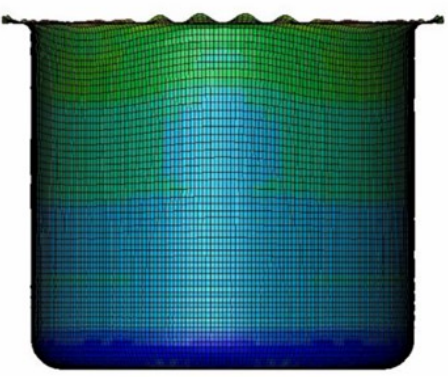

Толщина, мм

1,2010

1,1390

1,0770

1,0150

0,9532

0,8912

0,8291

0,7671

0,7050

0,6430

0,5809

Рис. 5. Деформированное состояние (а) и поле толщин в мм (б) стакана после этапа II

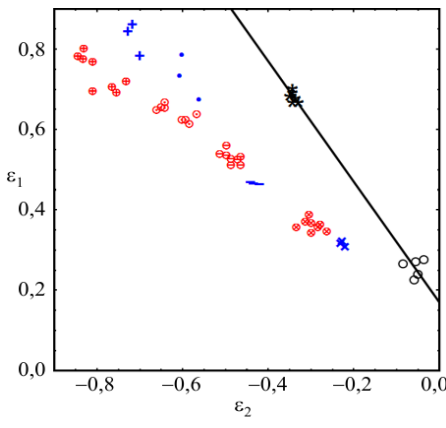

$a$

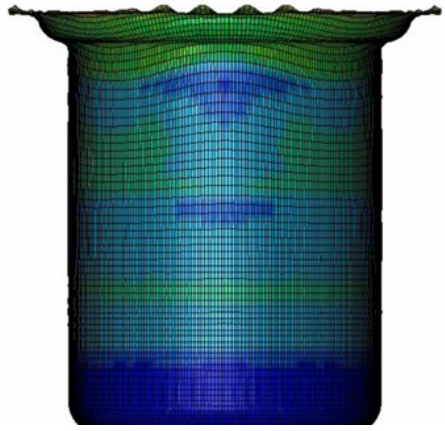

$\sigma$

Рис. 6. Деформированное состояние (а) и поле толщин в мм (б) стакана после этапа III

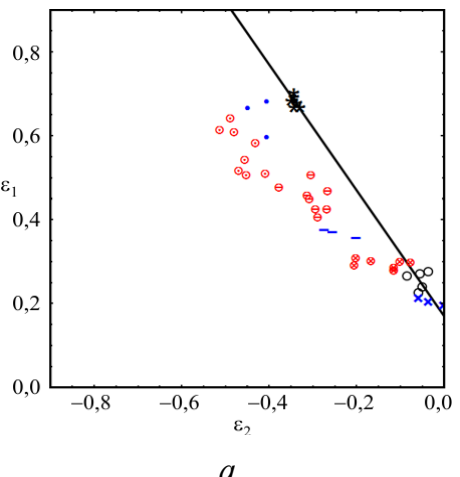

$a$

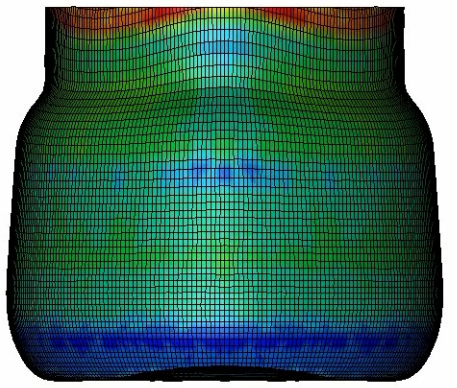

Толшина, мм 0,9684 0,9258 0,8832 0,8406 0,7980 0,7554 0,7128 0,6702 0,6277 0,5851 0,5425

Рис. 7. Деформированное состояние (а) и поле толщин в мм (б) изделия после раздачи без промежуточного отжига 
(в шкале логарифмических деформаций: $\varepsilon_{1}$ - продольной и $\varepsilon_{2}-$ окружной).

Для экспериментального определения деформаций в контрольных точках четыре круговых исходных заготовки с помощью лазерной гравировки покрывались сеткой координат (см. рис. 2). При этом радиальные полосы с шагом $45^{\circ}$ относительно направления прокатки и окружные полосы с шагом 20-24 мм наносились так, чтобы после любого этапа как минимум три полосы располагались на боковой поверхности изделия. Размер координатной ячейки составлял 2 мм. Далее первая заготовка подвергалась технологической операции I из последовательности рис. 1, вторая операциям I и II, третья - операциям I-III, четвертая - I-IV. По деформированной сетке в контрольных точках (точках пересечения координатных полос) для каждой из заготовок определялись главные логарифмические деформации.

Табл. 2 содержит значения $\delta$ - среднего расстояния от плоскости, на которой располагается дно сосуда, до деформированных круговых координатных полос. Численная информация о деформированном состоянии на каждом из этапов находилась в 8 контрольных точках соответствующего круга. На диаграммах деформаций (см. рис. 4-7) результатам эксперимента отвечают маркеры в кружках, численному расчету - маркеры без кружков. Там же изображена прямая линия предельных деформаций, аппроксимирующая экспериментальные данные одноосного рас-

Таблица 2

\section{Координаты маркеров контрольных точек} на исходной заготовке

\begin{tabular}{|l|l|c|c|c|c|}
\hline \multicolumn{2}{|l|}{ Координата (см. рис. 2), мм } & 108 & 130 & 150 & 174 \\
\hline $\begin{array}{l}\text { Маркер, эксперимент } \\
\text { (см. рис. 4-7) }\end{array}$ & $\bigotimes$ & $\ominus$ & $\odot$ & $\oplus$ \\
\hline $\begin{array}{l}\text { Маркер, расчет } \\
\text { (см. рис. 4-7) }\end{array}$ & $\times$ & - & $\cdot$ & + \\
\hline \multirow{4}{*}{$\delta$, мм } & этап I & - & 26 & 52 & 85 \\
\cline { 2 - 6 } & этап II & 24 & 53 & 84 & 126 \\
\cline { 2 - 6 } & этап III & 44 & 78 & 115 & 163 \\
\cline { 2 - 6 } & этап IV & 30 & 62 & 96 & - \\
\hline
\end{tabular}

тяжения (маркер *) и плоской деформации (маркер о), полученные ранее в $[12,14]$.

Расчет этапа I с увеличенным зазором между краями заготовки и прижимом (условия плоского напряженного состояния) демонстрирует образование четырех фестонов на краях стакана и гофр на всей его верхней части, что подтверждается экспериментом. Расчет с нулевым зазором обеспечивает плоское деформированное состояние, полностью подавляет гофрообразование и уменьшает размер фестонов, но ведет к неоднородности распределения толщины листа и главных деформаций по окружной координате в верхней части стакана (см. рис. 4). Количество фестонов и их ориентация вдоль осей анизотропии в обоих случаях совпадает с оценками работ [17-19] с учетом характера анизотропии $r_{0} / r_{90}<1, \sigma_{0} / \sigma_{90}<1$ материала [12]. Неоднородность распределения деформации по кругу в верхней части стакана после операции I и ее эволюция в операциях II-III демонстрируется на рис. 4-6 разбросом данных расчета и эксперимента (см. маркеры «Х»и $\otimes$ соответственно). Эта неоднородность сначала увеличивается, затем, по мере вытяжки, слегка уменьшается.

На рис. 7 показано состояние сосуда после этапа раздачи без предварительного отжига. Некоторое расхождение расчетных и экспериментальных данных о деформированном состоянии в контрольных точках наследуется с предыдущего этапа. Расчет прогнозирует, что деформированное состояние сосуда достигает предельного уровня на полосе боковой поверхности вблизи его максимального диаметра (в окрестности маркера $\bigoplus$ ), что подтверждается экспериментом (см. рис. 3). В предыдущей работе [14] рассчитывалось ссаживание стакана после предварительного отжига, который задавался обнулением внутренней переменной накопленной деформации во всем объеме материала. Деформированное состояние заготовки после завершения этого этапа слабо отличалось от результата без отжига, что под- 
тверждается экспериментом. Причина фактического разрушения заготовки, сопровождающего операцию без отжига, будет обсуждаться в следующем разделе.

На рис. 8 приведены траектории деформирования в контрольных точках боковой поверхности сосуда. Данные на диаграммах $a-8$ соответствуют направлению прокатки, а на г-направлениям относительно направления прокатки $0^{\circ}$ (тонкая линия) и $45^{\circ}$ (толстая линия). Рассчитанные кривые практически проходят через экспериментальные точки (отмечены, соответственно, разноразмерными маркерами $\oplus$ и $\bigoplus$ ). Различие данных на рис. 8,2 демонстрирует степень развития неоднородности деформации, вызванной пластической анизотропией. Состояние материала в контрольных точках с маркером $\bigoplus$ оказывается довольно близко к линии предельных сдвиговых деформаций, но на этапе раздачи эти точки располагаются в области горловины, которая практически не деформируется. Расчет и эксперимент

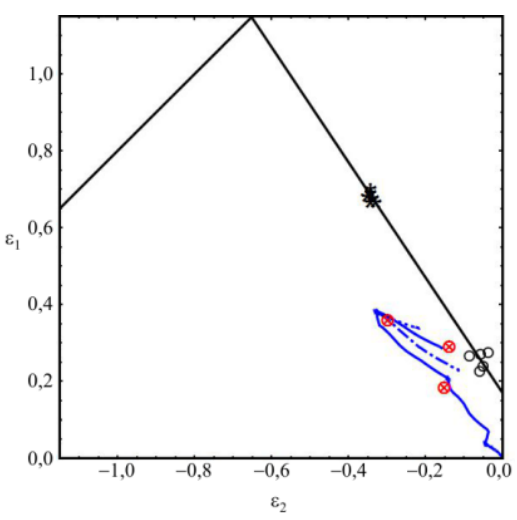

$a$

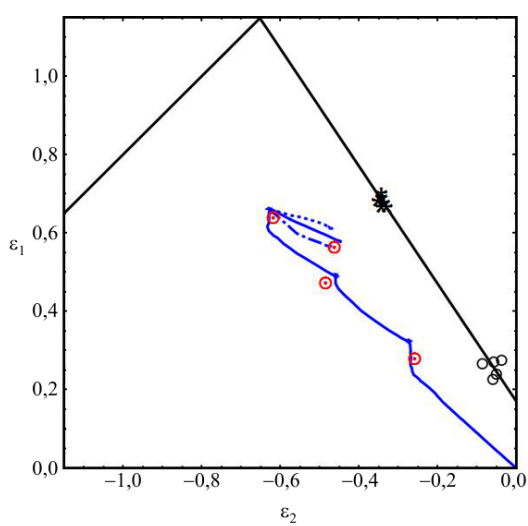

Рис. 8. Траектории деформирования в контрольных точках во время этапов I-IV технологического процесса показывают, что каждому этапу вытяжки стакана отвечает почти пропорциональное изменение компонент деформаций (простое нагружение), а этап раздачи сопровождается изломом траектории приблизительно на $150^{\circ}$. Эта особенность может выводить принятые модели пластичности и разрушения листовой стали за пределы областей их применимости.

На рис. 8 также приведены траектории деформаций, соответствующие альтернативным технологиям, применяемым на этапе IV штамповки чайника: раздаче полиуретаном с вертикальным поддавливанием кромки стакана (см. штрихпунктирные линии) и гидроформовке (пунктирные линии). В первом случае контролировалась скорость вертикального движения кромки, относительного поверхности штампа, во втором - гидростатическое давление. На состоянии материала обе технологии сказываются скорее благоприятно, но экспериментальное подтверждение этого отсутствует.
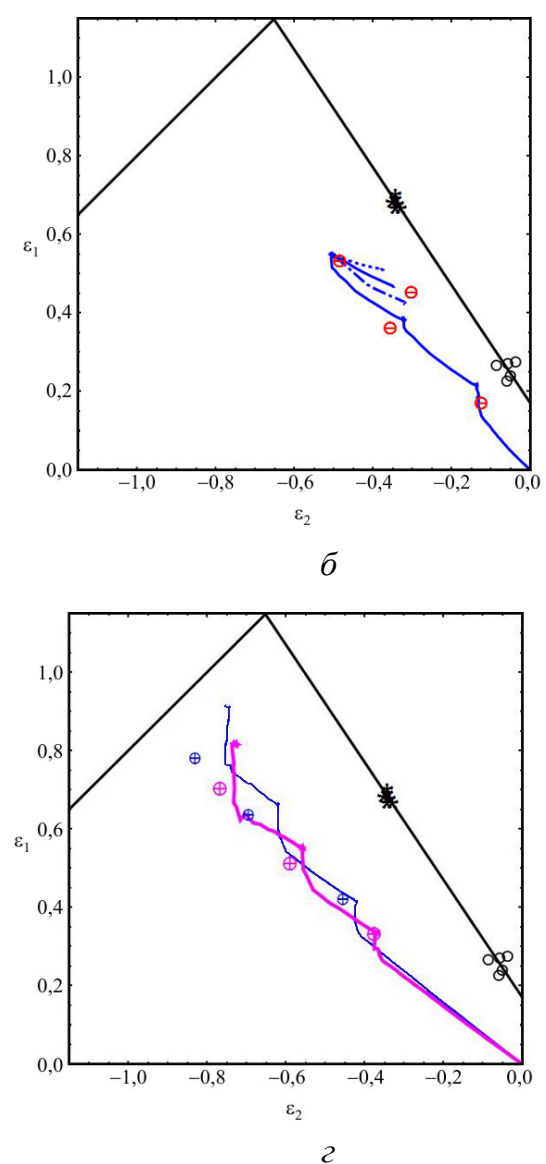

2 
Операция ссаживания горловины сосуда, выполняемая обкаткой роликом на специальной оправке (см. рис. 1), моделировалась как с учетом технологической наследственности, так и без него. Для формирования горловины (см. рис. 2) задавалась программа обкатки, включающая четыре вертикальных прохода вдоль горловины, первые три из которых сопровождаются двадцатью, а последний - десятью оборотами ролика вокруг горловины за один проход. На рис. 9 приведены схема конечно-элементной дискретизации оболочки и технологической оснастки и поле толщин горловины сосуда, образовавшееся в результате выполнения операции (показан результат упрощенного расчета без учета пластической анизотропии, накопленных деформаций и толщин оболочки, сформированных предыдущими этапами; расчет проведен с целью анализа траекторий деформаций и устойчивости процесса к образованию дефектов геометрии). Рис. 10 содержит траектории деформаций в контрольных точках боковой поверхности $a$, а также финальные толщины оболочки в этих точ-

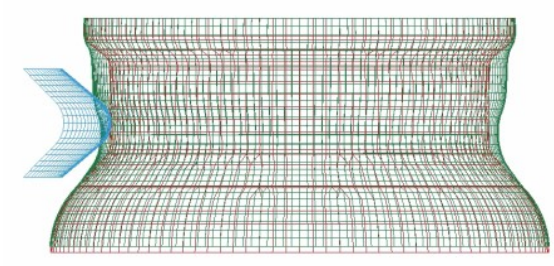

$a$ ках в, показывающие большее утонение стенки сосуда в зонах переходов диаметров в операции ссаживания. Близость этих зон к предельному состоянию подтверждается известными случаями срезания горловины инструментом. Расчет при условии однородно распределенного деформационного упрочнения дает результаты, практически неотличимые от приведенных на рис. 10. Расчет с полным учетом технологической наследственности оказывается неустойчивым из-за неравножесткости заготовки вследствие неоднородности распределения толщин по угловой координате.

Оценка технологического процесса по близости к предельному состоянию. Качество заготовки в результате выполнения каждого этапа и технологического процесса штамповки тонкостенного сосуда в целом оценивается с помощью меры близости состояния материала в контрольных точках боковой поверхности заготовки к предельным деформациям на диаграмме $\left\{\varepsilon_{1} \geq 0, \varepsilon_{2} \leq 0\right\}$. На этой диаграмме выделяют линию предельных деформаций [1], при достижении которой недопустимо раз-

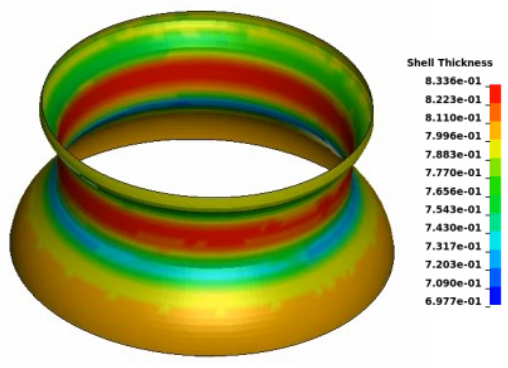

$\sigma$

Рис. 9. Ссаживание горловины на оправке: текущая конечно-элементная сетка (а) и поле толщин в мм в финальном состоянии (б)

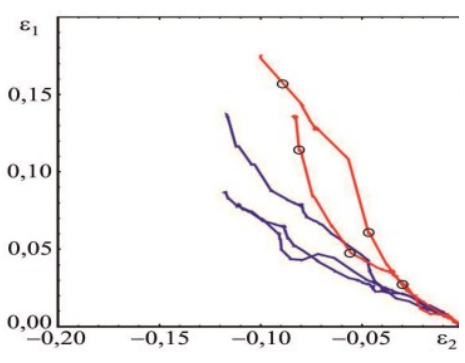

$a$

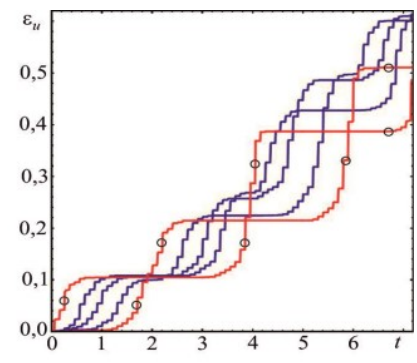

6

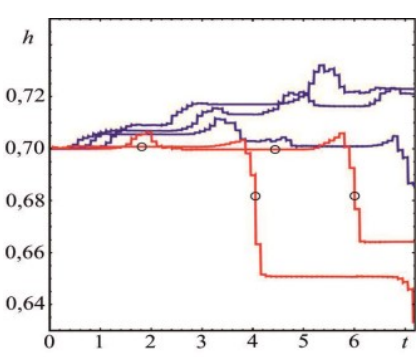

6

Рис. 10. Ссаживание горловины на оправке: траектории деформирования (а), изменение накопленной деформации (б), толщины в мм (в) в контрольных точках горловины: в середине (немаркированные кривые) и вблизи краев (кривые с маркерами) 
вивается утонение стенки (на плоском образце образуется шейка). Положение этой линии для используемой стали определено в предыдущих работах $[12,14,15]$ по данным испытаний (см. рис. 4-8), в которых обеспечивались одноосное растяжение и состояние, близкое к плоской деформации. Достижение линии предельных сдвиговых деформаций, введенной авторами работы [20], означает пластическое разрушение по сдвиговой моде. Эта линия также показана схематически на рис. 8. Следует отметить, что вблизи оси $\varepsilon_{2}$ на диаграмме деформаций находятся состояния, опасные с точки зрения гофрообразования [1], в которых сжимающая главная деформация критически превосходит растягивающую. Дефекты, возникающие в рассматриваемой технологии производства чайника без промежуточного отжига, представляют собой полосы утонения вдоль линий максимальных касательных напряжений (см. рис. 3), то есть образуют картину, характерную для состояний, отвечающих области предельных деформаций.

Необходимо заметить, что экспериментальные точки для построения кривой предельных деформаций получаются в результате панч-тестов и подобных испытаний, в которых реализуется простое нагружение $[1,4,21]$. Траектории деформации, характерные для рассматриваемого многоэтапного процесса (см. рис. 8), являются непропорциональными, поскольку после излома они соответствуют активному нагружению (упругие деформации ввиду своей малости на рисунке неразличимы). Существуют систематические эксперименты Графа и Хосфорда $[22,23]$, свидетельствующие об эволюции кривой предельного деформирования в процессе простого нагружения, в ходе которого граница смещается внутрь безопасной области. В многоэтапных процессах это указывает на возможность проблем при раздаче, следующей после вытяжки осесимметричного сосуда. В то же время ссаживание после вытяжки практически продолжает пропорциональное нагружение и не прибли- жает материал боковой поверхности сосуда к предельному состоянию.

Наглядное изображение изменения деформированного состояния изделия и его близости к предельному состоянию позволяют сделать общие выводы о предпочтительных технологиях получения качественного изделия (в том числе и о возможности исключения промежуточного отжига). Менее опасной выглядит технология с последовательными этапами раздачи и ссаживания стакана большего диаметра после вытяжки. Диаметр стакана определяется степенью раздачи, не требующей восстановления ресурса пластичности предварительным отжигом. Повышение степени ссаживания горловины может быть достигнуто за счет увеличения числа проходов обкатки роликом. Меньшая степень вытяжки стакана сохраняет его равножесткость по окружной координате и, следовательно, - устойчивость процесса ссаживания.

\section{Заключение}

С использованием численного расчета последовательных этапов вытяжки, раздачи и ссаживания тонкостенного осесимметричного сосуда построены траектории деформации его боковой поверхности в контрольных точках. Установлено, что траектории деформаций материала приближаются к предельному состоянию в основном во время раздачи заготовки, следующей после вытяжки. Такая смена операций ведет к резкому излому траекторий деформаций и смещению предельных деформаций внутрь безопасной зоны, что было показано в экспериментах Графа и Хосфорда. Отмечено, что операция ссаживания (редукции диаметра осесимметричного сосуда) после вытяжки практически соответствует продолжению траектории деформирования точки материала в прежнем направлении. Поскольку этими операциями достигается получение заданной формы сосуда, обеспечить его можно сочетанием меньшей степени раздачи и большей степени ссаживания заготовки с 
увеличенным диаметром. Позволит ли оптимальный подбор параметров альтернативной технологии исключить операцию отжига - вопрос, требующий обязательного подтверждения натурными испытаниями. Сказанное является общим рассуждением, основывающимся на ана- лизе эволюции деформированного состояния изделия в ходе каждой из последовательных операций многоэтапного процесса штамповки и, безусловно, нуждается в самостоятельном исследовании. Основные результаты по проекту опубликованы в работах $[4,12,14,24,25]$.

\section{Библиографический список}

1. Banabic D. Sheet metal forming processes. Constitutive modelling and numerical simulation. Springer, 2010. $-301 \mathrm{p}$.

2. Ни P., Ma N., Liu L., Zhu Y. Theories, methods and numerical technology of sheet metal cold and hot forming. Analysis, simulation and engineering applications. Springer, 2013. $-210 \mathrm{p}$.

3. Bruschi S., Altan T., Banabic D. [et al.] Testing and modelling of material behaviour and formability in sheet metal forming // CIRP Annals. - 2014. - Vol. 63. - P. 727-749.

4. Келлер И.Э., Петухов Д.С., Казанщев А.В., Трофимов В.Н. Диаграмма предельных деформаций при горячей листовой штамповке металлов. Обзор моделей материала, критериев вязкого разрушения и стандартных испытаний // Вестн. Самарского гос. техн. ун-та. Сер. Физ.-мат. науки. - 2018. - Т. 22. - № 3. - C. 447-486.

5. Bariani P.F., Dal Negro T., Bruschi S. Testing and modelling of material response to deformation in bulk metal forming // CIRP Annals. - 2004. - Vol. 53. - P. 573-595.

6. Kim B.J., Van Tyne C.J., Lee M.Y., Moon Y.H. Finite element analysis and experimental confirmation of warm hydroforming process for aluminum alloy // J. Mater. Process. Tech. - 2007. - Vol. 187-188. - P. 296-299.

7. Shafaat M.A., Abbasi M., Ketabchi M. Investigation into wall wrinkling in deep drawing process of conical cups // J. Mater. Process. Tech. - 2011. - Vol. 211. - P. 1783-1795.

8. Andrade F.X.C., Feucht M., Haufe A., Neukamm F. An incremental stress state dependent damage model for ductile failure prediction // Int. J. Fract. - 2016. - Vol. 200. - P. 127-150.

9. Neto D.M., Oliveira M.C., Dick R.E. [et al.] Numerical and experimental analysis of wrinkling during the cup drawing of an AA5042 aluminium alloy // Int. J. Mater. Form. - 2017. - Vol. 10. - P. 125-138.

10. Khan A.S., Huang S. Continuum theory of plasticity. John Wiley \& Sons, 1995. -421 p.

11. Barlat F., Brem J.C., Yoon J.W. [et al.] Plane stress yield function for aluminum alloy sheets - part 1: theory // Int. J. Plast. - 2003. - Vol. 19. - P. 1297-1319.

12. Адамов А.А., Келлер И.Э., Петухов Д.С. Экспериментальная идентификация законов пластичности и разрушения малоуглеродистой листовой стали для моделирования холодной штамповки // ППП. 2019. - T. 81. - № 2. - C. 202-211.

13. LS-DYNA ${ }^{\circledR}$ Keyword user's manual. Vol. II. Material models. Version R10.0. LSTC, 2017. - 1682 p.

14. Келлер И.Э., Казанщев А.В., Адамов А.А., Петухов Д.С. Моделирование многоэтапной холодной штамповки тонкостенного сосуда // ППП. - 2020. - Т. 82. - № 1. - С. 75-88.

15. Maker B.N., Zhu X. Input parameters for springback simulation using LS-DYNA // $6^{\text {th }}$ Int. LS-DYNA Conf. Detroit, USA, April, 2000. - 12 p.

16. Maker B.N., Zhu $X$. Input parameters for metal forming simulation using LS-DYNA // 3rd European LSDYNA Conf. Paris, France, June, 2001. - 10 p.

17. Hill R. A theory of the yielding and plastic flow of anisotropic metals // Proc. R. Soc. Lond. A. - 1948. Vol. 193. - P. 281-297.

18. Yoon J.W., Dick R.E., Barlat F. A new analytical theory for earing generated from anisotropic plasticity // Int. J. Plast. - 2011. - Vol. 27. - P. 1165-1184.

19. Chung K., Kim D., Park T. Analytical derivation of earing in circular cup drawing based on simple tension properties // Eur. J. Mech. Solid. - 2011. - Vol. 30. - P. 275-280.

20. Isik K., Silva M.B., Tekkaya A.E., Martins P.A.F. Formability limits by fracture in sheet metal forming // J. Mater. Process. Tech. 2014. - Vol. 214. - P. 1557-1565.

21. ISO 12004-2:2008. Metallic materials - Sheet and strip - Determination of forming-limit curves - Part 2: Determination of forming limit curves in the laboratory. International Organization for Standardization, 2008. $-27 \mathrm{p}$.

22. Graf A., Hosford W.F. Effect of changing strain paths on forming limit diagrams of Al 2008-T4 // MTA. 1993. - Vol. 24. - P. 2503-2512.

23. Graf A., Hosford W.F. The influence of strain-path changes on forming limit diagrams of Al $6111 \mathrm{~T} 4 / /$ Int. J. Mech. Sci. - 1994. - Vol. 36. - P. 897-910. 
24. Казанщев А.В., Келлер И.Э. Оценка многоэтапного технологического процесса холодной листовой штамповки тонкостенного сосуда с точки зрения предельных деформаций // Вычислительная механика сплошных сред. - 2020. - Т. 13. - № 2. - С.123-133.

25. Казанщев А.В., Келлер И.Э. Расчет многоэтапного процесса листовой штамповки тонкостенного сосуда и его оценка с точки зрения предельных деформаций // Вестник ЧГПУ им. И.Я. Яковлева. Сер.: Механика предельного состояния. - 2020. - № 4 (46). - С. 84-92.

\title{
THE LIMIT FORMING ESTIMATION OF MULTI-STAGE THIN-WALLED VESSEL COLD FORMING TECHNOLOGICAL PROCESS
}

\author{
I.E. Keller ${ }^{1,2}$, A.V. Kazantsev ${ }^{1}$, A.A. Adamov ${ }^{2}$, D.S. Petukhov ${ }^{1,2}$ \\ V.N. Trofimov ${ }^{1}$, A.N. Oborin ${ }^{3}$, S.B. Chugainov ${ }^{4}$ \\ ${ }^{1}$ Institute of Continuous Media Mechanics UB RAS \\ ${ }^{2}$ Perm National Research Polytechnic University \\ ${ }^{3}$ Lysva Plant of Enameled Cookware \\ ${ }^{4}$ Lysva Plant of Household Appliances
}

\section{For citation:}

Keller I.E., Kazantsev A.V., Adamov A.A., Petukhov D.S., Trofimov V.N., Oborin A.N., Chugainov S.B. The limit forming estimation of multi-stage thin-walled vessel cold forming technological process // Perm Federal Research Center Journal. 2021. - № 2. - P. 48-60. https://doi.org/10.7242/2658-705X/2021.2.2

The multi-stage technological process of cold stamping of a thin-walled vessel made of sheet steel was evaluated taking into account technological heredity. The quality of the product is evaluated by the distance of its deformed state from the limit states on the strain diagram. To calculate the process, the model of large plastic deformations of the anisotropic shell is used, taking into account the dynamics and contact interactions with the tool, which was numerically implemented in the LS-DYNA ${ }^{\circledR}$ package. Models of plastic flow of an anisotropic sheet, associated with the Barlat criterion Yld 2000-2d, with the power law of isotropic strain hardening, the Peng - Landel potential of nonlinear elastic behavior of a polyurethane die, and the Coulomb friction law of contact interaction of the product with the tool are used from the package library. These models constants for low-carbon sheet steel DC04EK 0,7 $\mathrm{mm}$ and polyurethane SKU-PFL were identified from experimental data. The forming limit curve was built on the distortion of the coordinate grid near the zones of strain localization and failure of the vessel in the technological process without intermediate annealing and in the failure test under uniaxial tension. The features of the strain paths in the control points of the vessel at each stage of the technological process, including the sequence of operations of drawing, distributing and reduction, are studied. The path calculation was confirmed by an experiment using pressing equipment as a test. It is established that the operation of distributing the workpiece after its drawing quickly leads to the limit state and therefore requires a preliminary recovering the plasticity resource by annealing. There is a preference for forming the relief of the vessel by smaller degrees of distribution and greater degrees of reduction for elimination of the limit states and the operation of intermediate annealing.

Keywords: multi-stage technological process, cold sheet stamping, numerical calculation, anisotropic plasticity, limit strains, low-carbon steel, material model identification, experimental confirmation.

\section{Сведения об авторах}

Келлер Илья Эрнстович, доктор физико-математических наук, заведующий лабораторией нелинейной механики деформируемого твердого тела, Институт механики сплошных сред УрО РАН - филиал Пермского федерального исследовательского центра УрО РАН («ИМСС УрО РАН»), 614013, г. Пермь, ул. Академика Королева, 1; профессор, Пермский национальный исследовательский политехнический университет (ПНИПУ), 614990, г. Пермь, Комсомольский пр., 29; e-mail: kie@icmm.ru 
Казанцев Александр Владимирович, старший преподаватель кафедры динамики и прочности машин, ПНИПУ; e-mail: alexkazancev@bk.ru

Адамов Анатолий Арсагалеевич, доктор физико-математических наук, ведущий научный сотрудник лаборатории нелинейной механики деформируемого твердого тела, «ИМСС УрО РАН»; e-mail: adamov@icmm.ru

Петухов Дмитрий Сергеевич, ведущий инженер лаборатории нелинейной механики деформируемого твердого тела, ИМСС УрО РАН; старший преподаватель кафедры динамики и прочности машин, ПНИПУ; e-mail: petuhovds@mail.ru

Трофимов Виктор Николаевич, доктор технических наук, профессор кафедры динамики и прочности машин, ПНИПУ; e-mail: tvn_perm@mail.ru

Оборин Артем Николаевич, начальник отдела технического развития АО «Лысьвенский завод эмалированной посуды», 618909, Пермский край, г. Лысьва, ул. Металлистов, 1/20 стр. 5; e-mail: oborin-an@1pec.ru

Чугайнов Сергей Борисович, ведущий инженер-конструктор ООО «Лысьвенский завод бытовой техники», 618900, Пермский край, г. Лысьва, ул. Металлистов, 1; e-mail: chugainov@1zbt.ru 